\title{
The host galaxies of narrow-line Seyfert-1s: Evidence for bar-driven fueling
}

\author{
D. Michael Crenshaw ${ }^{1}$, Rajesh Deo ${ }^{1}$, S. B. Kraemer ${ }^{2}$ and J. R. Gabel ${ }^{3}$ \\ ${ }^{1}$ Department of Physics and Astronomy, Georgia State University, One Park Place South SE, \\ Suite 700, Atlanta, GA 30303, USA \\ ${ }^{2}$ Catholic University of America and Laboratory for Astronomy and Solar Physics, NASA \\ Goddard Space Flight Center, Code 681, Greenbelt, MD 20771, USA \\ ${ }^{3}$ University of Colorado, CASA, Boulder, CO 80309, USA
}

\begin{abstract}
We have studied the host-galaxy morphologies of narrow- and broad-line Seyfert 1 galaxies (NLS1s and BLS1s) based on broad-band optical images from the Hubble Space Telescope archives. We find that large-scale stellar bars, spanning $1-10 \mathrm{kpc}$ from the nucleus, are much more common in NLS1s than BLS1s. Furthermore, the fraction of NLS1 spirals that have bars increases with decreasing full-width at half-maximum (FWHM) of the broad component of $\mathrm{H} \beta$. This suggests a link between the large-scale bars, which can support high fueling rates to the inner kpc, and the high mass-accretion rates associated with the supermassive black holes in NLS1s. A preliminary examination of the inner $(<1 \mathrm{kpc})$ dust morphologies has revealed no significant differences between NLS1s and BLS1s, except that we confirm the previous finding that galaxies with large-scale bars have a much higher frequency of "grand design" nuclear dust spirals.
\end{abstract}

\section{Introduction}

The current paradigm is that, while AGN in general are powered by accretion of material onto a supermassive central black hole, NLS1s possess black holes of relatively modest mass $\left(\leqslant 10^{7} \mathrm{M}_{\odot}\right)$, that are accreting matter at or above their Eddington limits (Pounds, Done, \& Osborne 1995). Is there a connection between NLS1s and properties of their host galaxies to support the high accretion rates? Krongold, Dultzin-Hacyan, and Marziani (2001) examined host galaxies and environments, and found no statistical difference between the frequency of companion galaxies in NLS1s and BLS1s. Another possibility is a difference in their large-scale morphologies. One process for fueling AGN that has received considerable attention is gas inflow along a stellar bar (Simkin, Su, \& Schwarz 1980). Theoretical studies show that the gas can lose its angular momentum after encountering the gravitational potential of a bar and be transported inward (Shlosman, Begelman, \& Frank 1989). However, most observational studies in this area have found similar fractions of bars for Seyferts and normal galaxies (e.g., Hunt \& Malkan 1999). What happens if we look at subtypes, such as NLS1s and BLS1s?

\section{Observations and Results}

Our sample contains primarily the HST broad-band images of Seyfert 1 galaxies obtained by Malkan, Gorjian, \& Tam (1998). This is a uniform sample of 91 Seyfert 1 galaxies at $\mathrm{z} \leqslant 0.035$ observed with the Wide Field Planetary Camera 2 (WFPC2) through the F606W filter. The number of NLS1s in this sample is small (13); so we have included 6 additional NLS1s from Véron-Cetty et al. (2001) that have WFPC2 broad-band optical images. We retrieved all of the WFPC2 images from the HST archives, to display 
Figure 1. HST/WFPC2 image of MCG 6-26-12, which harbors a NLS1. The field of view is $34^{\prime \prime} \times 34^{\prime \prime}$, which corresponds to $21 \mathrm{kpc} \times 21 \mathrm{kpc}$.

a larger field of view $\left(37^{\prime \prime} \times 37^{\prime \prime}\right.$, the projected size of the PC) than the images published (mostly $9^{\prime \prime} \times 9^{\prime \prime}$ ) in Malkan et al. (1998). To ensure objectivity, we scrambled the order of the HST images and the coauthors independently classified their morphologies without knowledge of Seyfert 1 subclass (NLS1 or BLS1) or the name of the galaxy. We classified the galaxies into one of 6 major groups: S (spiral), SB (spiral with a noticeable bar), E (elliptical), I (irregular), P (point-souce, only the active nucleus is visible), or ? (uncertain). An example of a spiral with a strong stellar bar is shown in Figure 1.

We have classified $91 \%$ of the galaxies in the sample as spirals, and of the spirals, we have classified $33 \%$ as barred. The latter is consistent with percentages from studies that have identified strong bars in optical images of both active and normal spiral galaxies (Mulchaey et al. 1997). However, most of the NLS1 galaxies are barred spirals, and most of the BLS1 galaxies are normal spirals. Quantitatively, if we consider the 84 spirals in this sample, then $65 \%(11 / 17)$ of the NLS1 spirals have bars, whereas only $25 \%(17 / 67)$ of the BLS1 spirals have bars. If we consider only the Malkan et al. sample, then $64 \%$ $(7 / 11)$ of the NLS1 spirals have bars, which is essentially identical to the result for the full sample.

Figure 1 shows histograms of the fraction of spirals that have bars as a function of the FWHM $(\mathrm{H} \beta)$ on a log scale. The shaded regions show a clear trend of decreasing fraction of bars with increasing FWHM for the entire sample. The trend continues through the three bins associated with NLS1s (FWHM $<2000 \mathrm{~km} \mathrm{~s}^{-1}$ ) to the first bin for the BLS1s. The fraction levels out thereafter, and is essentially constant for all BLS1s regardless of FHWM. Interestingly, the four NLS1 spirals in the full sample with the smallest FWHM 


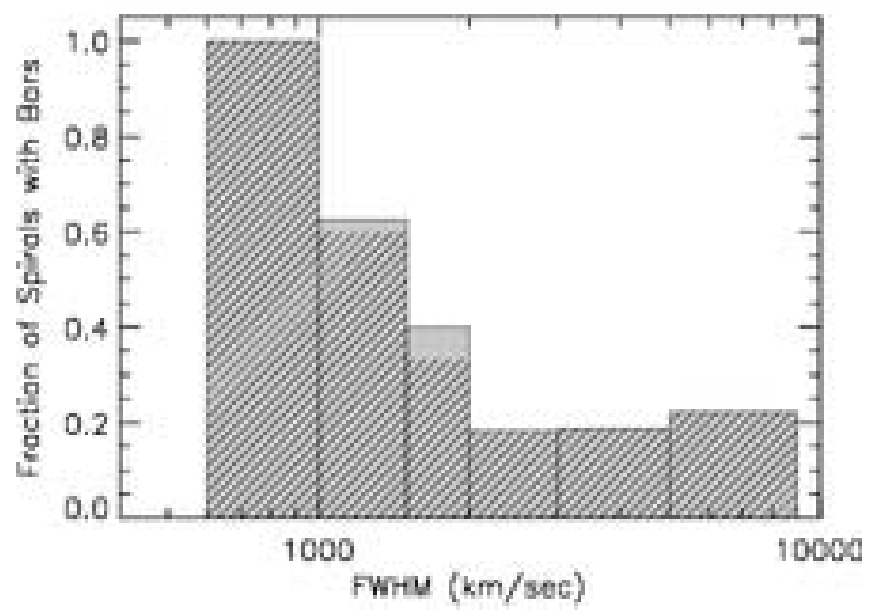

Figure 2. Histogram showing the fraction of Seyfert spirals with bars as a function of the full-width at half-maximum (FWHM) of the broad component of the $\mathrm{H} \beta$ emission line. The shaded region is for the full sample, whereas the hatched region is for the Malkan et al. (1998) subsample.

$\left(600-1000 \mathrm{~km} \mathrm{~s}^{-1}\right)$ all show bars. The hatched region in Figure 1 shows that the same trend occurs for the more uniform Malkan et al. subsample; there is only a slightly lower fraction of bars in NLS1s with widths in the range $1000-2000 \mathrm{~km} \mathrm{~s}^{-1}$ compared to the full sample.

\section{Discussion}

Our analysis of $H S T /$ WFPC2 images reveals that stellar bars are more common in NLS1s, particularly those with very narrow $\mathrm{H} \beta$ profiles, compared to BLS1s, as discussed in more detail in Crenshaw et al. (2003). This suggests a link between the high fueling rates that can be provided by the bars and the high accretion rates commonly associated with NLS1s. These large-scale stellar bars often extend down to $1 \mathrm{kpc}$ from the nuclei of these galaxies. Inside a radius of $\sim 1 \mathrm{kpc}$, nuclear dust spirals, and in a few cases dust bars, are likely to be directly responsible for transporting fuel to the active nucleus. However, the exact connection between large-scale bars, inner dust spirals and bars, and accretion rates in the nucleus is unclear. We have created preliminary structure maps (Pogge \& Martini 2002) of the inner ( $\leqslant 1 \mathrm{kpc}$ ) regions of this sample, to study the nuclear dust morphology. As found in previous studies (Martini et al. 2003), nuclear dust spirals are present in most objects, and grand-design dust spirals are much more common in galaxies with large stellar bars. However, there is no other clear evidence (yet) that NLS1s have different nuclear dust morphologies than BLS1s.

\section{References}

Crenshaw, D. M., Kraemer, S. B., \& Gabel, J. R. 2003, AJ, 126, 1690

Hunt, L. K., \& Malkan, M. A. 1999, ApJ, 516, 660

Kronggold, Y., Dultzin-Hacyan, D., \& Marziani, P. 2001, AJ, 121, 702

Malkan, M. A., Gorjian, V., \& Tam, R. 1998, ApJS, 117, 25

Martini, P., Regan, M. W., Mulchaey, J. S., \& Pogge, R. W. 2003, ApJ, 589, 774

Pogge, R. W. \& Martini, P. 2002, ApJ, 569, 624 
Pounds, K. A., Done, C., \& Osborne, J. P. 1995, MNRAS, 277, L5

Shlosman, I., Begelman, M. C., \& Frank, J. 1990, Nature, 345, 679

Simkin, S. M., Su, H. J., \& Schwarz, M. P. 1980, ApJ, 237, 404

Véron-Cetty, M.-P., Véron, P., \& Gonçalves, A. C. 2001, A\&A, 372, 730 